\title{
ANALYSIS OF THE MEAN FIRST LOOPING TIME OF A ROD-POLYMER*
}

\author{
A. AMITAI $^{\dagger}$, I. KUPKA ${ }^{\ddagger}$ AND D. HOLCMAN ${ }^{\dagger}$
}

\begin{abstract}
We present a new approach to investigating the dynamics of loop formation in a very crude polymer model and estimate the mean first time for the two ends to meet. This time depends on the number of monomers $N$. We obtain analytical formulas when $N=3,4$ in dimension two and an asymptotic formula when $N$ is large. Our analysis is confirmed by stochastic simulations.
\end{abstract}

Key words. mean first passage time, polymer, asymptotic computation, freely jointed chain, looping

AMS subject classifications. 60G20, 60H30, 35J25, 35P15

DOI. $10.1137 / 110842624$

1. Introduction. Polymer looping has been studied both theoretically $[26,25$, $19,5]$ and numerically [10] using the Rouse model [4]. In that model, a polymer is modeled as a collection of monomers connected by springs [4]. Using heuristic arguments with reduced models, when the polymer moves in three dimensions, the mean time for a loop to form has been estimated numerically and is approximated by a power law in the number of monomers $N$.

We study here the properties of looping polymers composed of $N$ segments. In dimension two, the angle between the segments is a random variable and we shall estimate the mean time for the two ends to meet. We use a crude polymer model consisting of a freely jointed chain [4, p. 8]. More specifically, the polymer is an ordered chain of monomers (points) in the two dimensional plane, such that each monomer is connected to its two neighbors by a rigid bond, all these bonds having the same length $b$. The monomer motion is governed by independent and identically distributed Brownian motions. As we will see here, this dynamics results in long range correlations between monomers, which is different from Rouse-type dynamics. However, the present analysis captures much of the complexity of the looping problem, and the method we use can be adapted to other polymer models such as the Rouse model.

When the first monomer is fixed at the origin (which we can always assume using the translation invariance of the problem), we compute the mean time for the free moving end to encounter the fixed one. Because the probability of such an event is zero, we shall estimate the mean first time for the moving end to enter the disk of radius $\epsilon b$, centered at the fixed end (Figure 1). Here $\epsilon \ll 1$ is a dimensionless parameter. The average time $\left\langle\tau_{\epsilon}\right\rangle$ is called the mean first looping time (MFLT).

Our goal is to investigate the asymptotic behavior of $\left\langle\tau_{\epsilon}\right\rangle$ when the radius $\epsilon$ goes to zero. The main analytical results concern the planar case, although we will show some simulations for the three dimensional space. First, we shall introduce the

${ }^{*}$ Received by the editors July 28, 2011; accepted for publication (in revised form) January 9, 2012; published electronically June 7, 2012.

http://www.siam.org/journals/mms/10-2/84262.html

${ }^{\dagger}$ Institute for Biology, IBENS, Group of Computational Biology and Applied Mathematics, École Normale Supérieure, 75005 Paris, France (amitai@biologie.ens.fr, holcman@biologie.ens.fr). The third author's research was supported by an ERC Starting Grant.

${ }^{\ddagger}$ Institut de Mathématique de Jussieu, Université Paris VI, 75005 Paris, France (prukupka@ aol.com). 
polymer model used here, and then we will state the constraints related to generating the loop for the first time in geometrical terms. Moreover, we compute the MFLT for $N=2,3,4$ asymptotically when $\epsilon$ is small. These computations are based on eigenvalue expansions associated with the Laplace operator on a domain with a regular boundary [2]. Unfortunately, although the method can be applied to arbitrary long polymers, the asymptotic computations cannot be performed. Thus, we approximate the end point dynamics by a new stochastic equation. When the chain is initially stretched, we obtain that for $N \gg 1$, the MFLT is given by

$$
\left\langle\tau_{\epsilon}\right\rangle=\frac{1}{D} \log \left(\frac{N}{\epsilon}\right)+\mathcal{O}_{\epsilon, \mathcal{N}}(1)
$$

where $\mathcal{O}_{\epsilon, \mathcal{N}}(1)$ means that the next term is of order 1 for small $\epsilon$ and large $N . D$ (in the unit of $s^{-1}$ ) is the diffusion constant of the typical Brownian motions governing the angles between each bond and the $x$-axis, while $N$ is the total number of bonds. When the initial configuration is at equilibrium, we obtain that

$$
\left\langle\tau_{\epsilon}\right\rangle=\frac{1}{D} \log \left(\frac{\sqrt{2 N}}{\epsilon}\right)+\mathcal{O}_{\epsilon, \mathcal{N}}(1),
$$

showing that the dynamics has a long memory, keeping track of the initial configuration. Our analysis cannot be extended easily to the three dimensional case, and we present only some numerical simulations, suggesting that the MFLT behaves like

$$
\left\langle\tau_{\epsilon}\right\rangle \approx C_{3} \frac{N^{\alpha}}{\epsilon D}
$$

where $C_{3}$ is a constant and $\alpha=0.48 \pm 0.02$.

\section{Model and formulation.}

2.1. Rod-polymer dynamics. A rod-polymer model is an ordered collection of $N+1$ monomers (points) with positions $\left(\boldsymbol{R}_{0}, \boldsymbol{R}_{1}, \ldots, \boldsymbol{R}_{N}\right)$ in the complex plane, such that $\left|\boldsymbol{R}_{i}-\boldsymbol{R}_{i-1}\right|=b(i=1, \ldots, N)$ while $\boldsymbol{R}_{0}=0$. The position at time $t$ of the $k$ th monomer is

$$
\boldsymbol{R}_{k}(t)=\boldsymbol{R}_{k-1}(t)+b \exp \left(i \theta_{k}(t)\right)
$$

where $\theta_{k}(t) \in[0,2 \pi]$ is the angle between the $x$-axis and $\boldsymbol{R}_{k}(t)-\boldsymbol{R}_{k-1}(t)$. Thus, the position of the $k$-monomer at time $t$ is given by

$$
\boldsymbol{R}_{k}(t)=b \sum_{j=1}^{k} \exp \left(i \theta_{j}(t)\right) .
$$

The motion of the chain is governed by that of the angles $\theta_{1}, \theta_{2}, \ldots, \theta_{N}$ which are independent Brownian variables on the circle, satisfying the stochastic equation

$$
d \theta_{j}=\sqrt{2 D} d B_{j}
$$

where $D$ is the rotational diffusion coefficient, and $B_{i}$ are Brownian variables on a circle of radius 1 with variance 1 . The dynamics of the rod-polymer end is represented by the mapping $P(t)=\left(e^{i \theta_{1}(t)}, \ldots, e^{i \theta_{N}(t)}\right)$ on the $N$-dimensional torus and by

$$
\begin{aligned}
\Psi: T_{N} & \rightarrow \mathbb{R}^{2}, \\
\left(\theta_{1}, \ldots, \theta_{N}\right) & \rightarrow \sum_{k=1}^{N} \exp \left(i \theta_{k}\right) .
\end{aligned}
$$


Thus

$$
\boldsymbol{R}_{N}(t)=b \Psi(P(t)) .
$$

2.2. The closed configuration space. Estimating the mean time $\left\langle\tau_{\epsilon}\right\rangle$ is equivalent to studying the Brownian dynamics of the point $P(t)=\left(e^{i \theta_{1}(t)}, \ldots, e^{i \theta_{N}(t)}\right)$ on the $N$-torus $T_{N}$ and evaluating the MFLT to the manifold

$$
B_{\epsilon}=\left\{\left(\theta_{1}, \ldots, \theta_{N}\right) \in T_{N} \text { such that }\left|\sum_{k=1}^{N} \exp \left(i \theta_{k}\right)\right|^{2} \leq \epsilon^{2}\right\} .
$$

The manifold $B_{\epsilon}$ is a closed neighborhood of an $(N-2)$ dimensional real analytic set $B_{0}$ given by (10), taking $\epsilon=0 . B_{0}$ is of codimension 2 because it is the set of points defined by the two independent equations

$$
\sum_{k=1}^{N} \sin \theta_{k}=0 \quad \text { and } \quad \sum_{k=1}^{N} \cos \theta_{k}=0
$$

The boundary $\partial B_{\epsilon}$ is represented for $N=3$ and $N=4$ in Figure 2 for $\epsilon=0.2 b$. To understand the nature of $\partial B_{\epsilon}$, we shall focus on $\epsilon=0$. There are indeed many closed configurations, some of which are depicted in Figure 1: for $N=3$, the set of all closed chains (Figure 1(B)) is an equilateral triangle with one vertex at zero, and this set is invariant under rotations. Under this action, it consists of two orbits. Any reflection (preserving zero) permutes these two orbits. For $N=4$, there are three types of configurations (Figure 1(C)): a lozenge and two nonisomorphic configurations. These structures are connected by continuous deformations, which allow us to characterize the boundary $\partial B_{\epsilon}$ : it is made of blobs, connected by narrow structures (Figure 2(b)). The description of $B_{0}$ for large $N$ is quite difficult, but surprisingly, as we will see, the mean time for the chain to close depends only on its volume when $\epsilon$ goes to zero.

3. Computing the MFLT $\left\langle\tau_{\epsilon}\right\rangle$. The MFLT starting from an initial position $P$ is the solution of the boundary value problem [12]

$$
\begin{aligned}
D \Delta_{N} u_{\epsilon}(P) & =-1 \quad \text { for } P \in T_{N}-B_{\epsilon}, \\
u_{\epsilon}(P) & =0 \quad \text { for } P \in \partial B_{\epsilon},
\end{aligned}
$$

where

$$
\Delta_{N}=\sum_{k=1}^{N} \frac{\partial^{2}}{\partial \theta_{k}^{2}}
$$

Our purpose is to give an asymptotic estimation of the uniform average of $u_{\epsilon}(P)$ for $\epsilon$ small. Unfortunately, this problem does not fall into the class of a narrow escape problem $[23,22,24,7,14,15,16,11]$, where in order to escape, a Brownian particle has to find a small hole located on the boundary of domain.

The MFLT is the average of $u_{\epsilon}$ over a uniform distribution:

$$
\left\langle\tau_{\epsilon}\right\rangle=\frac{1}{\left|T_{N}-B_{\epsilon}\right|} \int_{T_{N}-B_{\epsilon}} u_{\epsilon}(P) d V_{P}
$$



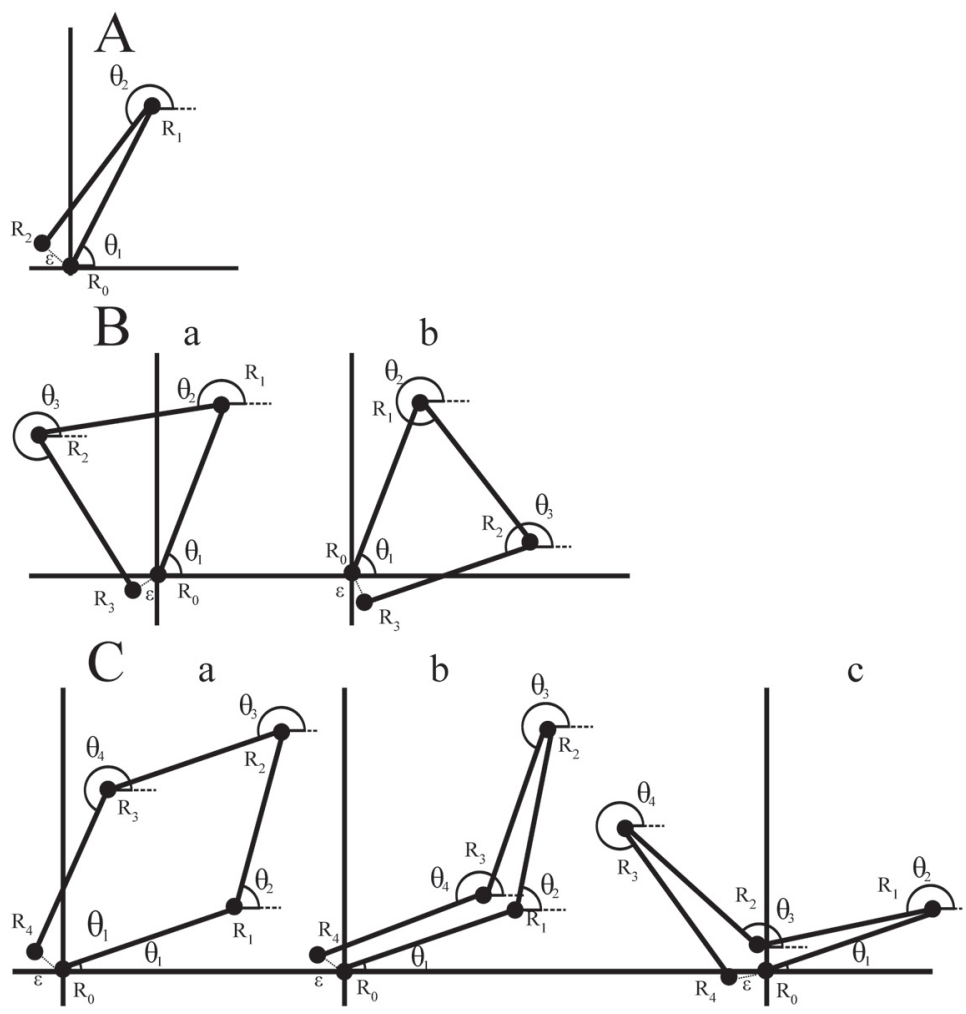

FIG. 1. Schematic representation of polymer loops for different lengths. Monomers positions are $\boldsymbol{R}_{k}$ while $\boldsymbol{R}_{0}$ is fixed at zero. $\theta_{k}$ is the angle between $\boldsymbol{R}_{k}-\boldsymbol{R}_{k-1}$ and the positive direction of the $x$-axis. $\epsilon$ is the absorption distance. (A) two bond polymer $(N=2)$ : there is only one closed configuration (up to rotation). (B) three bonds polymer $(N=3)$, showing that the only closed configuration is an equilateral triangle (the loop can be either clockwise or counterclockwise). (C) four bond polymer $(N=4)$, showing three possible closed configurations.
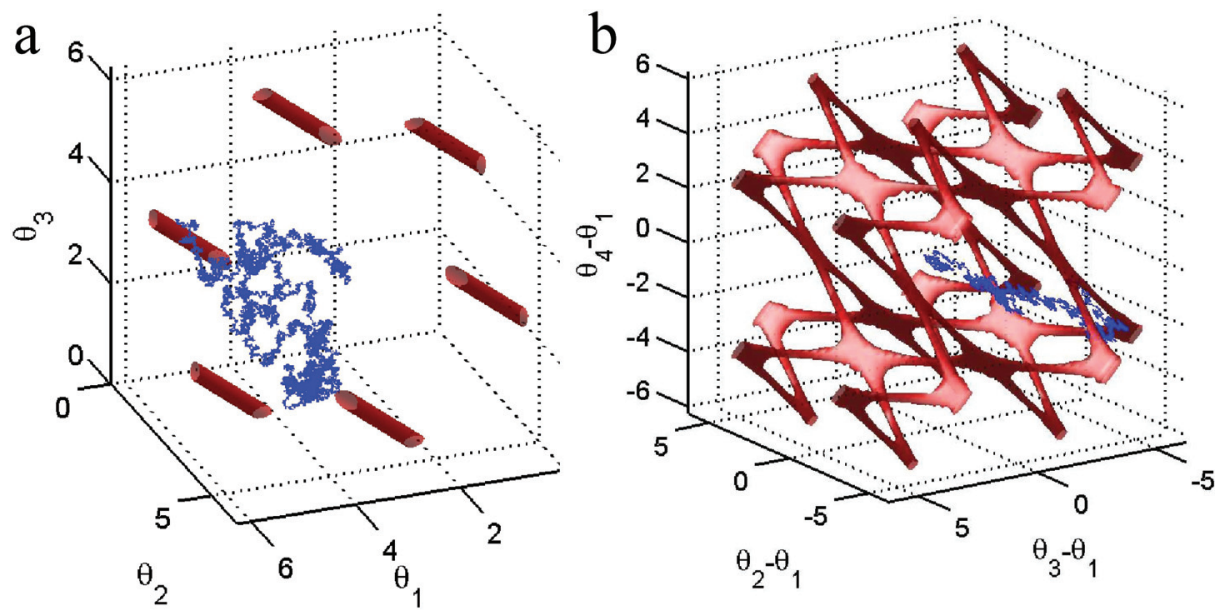

FIG. 2. The boundary of $B_{\epsilon}$ for a rod-polymer. (a) for $N=3$ and $\epsilon=0.2$ in $[0,2 \pi] *[0,2 \pi] *$ $[0,2 \pi]$. Although (a) show 6 disconnected, there are only two of them in a unitary torus. A Brownian trajectory (blue) in the angle space moves until it is absorbed. (b) for $N=4$ and $\epsilon=0.2 b$. The manifold consists of blobs connected by a narrow tube, corresponding to deformation of loops. 
where $\left|T_{N}-B_{\epsilon}\right|$ is the volume of $T_{N}-B_{\epsilon}$. When $\epsilon \ll 1,\left\langle\tau_{\epsilon}\right\rangle$ can be approximated by the first eigenvalue problem associated with problem (12). Indeed,

$$
\left\langle\tau_{\epsilon}\right\rangle=\int_{T_{N}-B_{\epsilon}} \int_{0}^{\infty} p(P, t) d t d V_{P},
$$

where $p$ is the solution of

$$
\begin{aligned}
\frac{\partial p}{\partial t} & =D \Delta_{N} p, \\
p(P, 0) & =\frac{1}{\left|T_{N}\right|}, \\
p(P, t) & =0 \text { for } P \in \partial B_{\epsilon} .
\end{aligned}
$$

By expanding $p$ over the eigenfunction basis $w_{\epsilon, k}$ of the Laplace operator on $T_{N}-B_{\epsilon}$ with a Dirichlet condition, we obtain that

$$
p(P, t)=\sum_{k=0} \frac{c_{k}}{\left|T_{N}\right|} e^{-D \lambda_{\epsilon, k} t} w_{\epsilon, k}(P),
$$

where $\lambda_{\epsilon, k}$ are the eigenvalues and $c_{k}=\int_{T_{N}-B_{\epsilon}} w_{\epsilon, k}$. Using (14), we obtain that

$$
\left\langle\tau_{\epsilon}\right\rangle=\sum_{k=0} \frac{c_{k}^{2}}{D\left|T_{N}\right| \lambda_{\epsilon, k}} .
$$

With a uniform initial distribution, we have $c_{0}^{2} /\left|T_{N}\right| \approx 1[11]$ and $c_{k}=\mathcal{O}(\epsilon)$ for $k \geq 1$. Finally,

$$
\left\langle\tau_{\epsilon}\right\rangle \approx \frac{1}{D \lambda_{\epsilon, 0}}
$$

We shall now further characterize the first eigenfunction. From conditions (11), the submanifold $B_{0}$ is of codimension 2 in a space of dimension $N . \lambda_{\epsilon, 0}$ is connected to the first eigenvalue $\lambda_{0}$ of the unperturbed problem (no boundary) [2] by

$$
\lambda_{\epsilon, 0} \approx \lambda_{0}+(2 \pi / \log (\epsilon)) \int_{B_{0}} w_{0}^{2} d V
$$

where $w_{0}$ is the first eigenfunction of $\Delta_{N}$ over the manifold $T_{N}$ and $d V$ is the volume induced on $B_{0}$ by the metrics $\sum d \theta_{i}^{2}$. This eigenfunction associated with $\lambda_{0}=0$ is constant and equals its normalized value

$$
w_{0}=\frac{1}{\sqrt{\left|T_{N}\right|}}=\frac{1}{(2 \pi)^{N / 2}} .
$$

Thus

$$
\lambda_{\epsilon, 0} \approx(2 \pi / \log (\epsilon)) \frac{\left|B_{0}\right|}{(2 \pi)^{N}} .
$$

Hence, the MFLT is approximated by its first order asymptotic term:

$$
\left\langle\tau_{\epsilon}\right\rangle \approx \frac{1}{2 \pi D} \frac{(2 \pi)^{N}}{\left|B_{0}\right|} \log \left(\frac{1}{\epsilon}\right) .
$$


Our goal is to determine the volume of $\left(\left|B_{0}\right|\right)$, which is a function of $N$. We will compute the volume in some specific cases. However, when $N$ is odd, $B_{0}$ is a manifold and when $N$ even, it has singularities (see Appendix A). This singular set is a finite union of circles, and thus the result of [2] can be applied. Finally, for a chain composed of $N$ monomers $\boldsymbol{R}_{N}=\boldsymbol{X}_{1}+\cdots+\boldsymbol{X}_{N}$ in dimension $n$, each $\boldsymbol{X}_{i}$ is moving on an $(n-1)$ dimensional sphere with radius $b$. Thus, the Brownian dynamics of $\boldsymbol{R}_{N}$ is that of the $N$-product of $(n-1)$ dimensional independent Brownian motions on $S^{n-1}$. Similar to the dimension 2 case, the analytic set $B_{0}$ is characterized by the condition

$$
\boldsymbol{X}_{1}+\cdots+\boldsymbol{X}_{N}=0
$$

showing that $B_{0}$ is of codimension $n$. Using the general result for the asymptotic expansion (in $\epsilon$ ) that relates the eigenfunctions and eigenvalues of the perturbed to the unperturbed problem [2], the $\operatorname{MFLT}\left\langle\tau_{\epsilon}\right\rangle$ is given by

$$
\left\langle\tau_{\epsilon}\right\rangle \approx \frac{1}{c_{n-1}(n-2) D} \frac{\left|S^{n-1}\right|}{\left|B_{0}\right|} \frac{1}{\epsilon^{n-2}} \approx \frac{1}{n(n-2) D} \frac{1}{\left|B_{0}\right|} \frac{1}{\epsilon^{n-2}},
$$

where $c_{n-1}$ is the area of the $n-1$ sphere $S^{n-1}$ and $\left|S^{n-1}\right|$ is the volume of the $n-1$ ball. To obtain the explicit dependency of the area of $B_{0}$ as a function of $N$ is in general extremely difficult to compute. We shall now present the computations for $N=2,3,4$ in the planar case.

3.1. Estimating the MFLT for $N=\mathbf{2}$. When $N=2, B_{\epsilon}$ is defined by the equation

$$
\left|\exp \left(i \theta_{1}\right)+\exp \left(i \theta_{2}\right)\right|^{2} \leq \epsilon^{2}
$$

that is,

$$
\cos \left(\theta_{1}-\theta_{2}\right) \leq \epsilon^{2} / 2-1
$$

$B_{0}$ as a subset of $T^{2}$ is given by

$$
\theta_{2}-\theta_{1}=\pi
$$

Setting $u \equiv \theta_{2}-\theta_{1}$, the analysis reduces to that of a standard Brownian motion with diffusion constant $2 D$ on the interval $[-\pi, \pi]$, with absorbing boundaries at $\pi-\epsilon$ and $-\pi+\epsilon$. The solution for an initial angle $\theta$ is $[1,12]$

$$
\left\langle\tau_{\epsilon}\right\rangle(\theta)=\frac{(\pi-\epsilon-\theta)(\theta+\pi-\epsilon)}{4 D} .
$$

Averaging over a uniform distribution gives

$$
\left\langle\tau_{\epsilon}\right\rangle \approx \frac{\pi^{2}}{6 D}
$$

and this result is independent of $\epsilon$. This case is very particular.

3.2. Estimating the MFLT for $\boldsymbol{N}=3$. We recall that

$$
B_{0}=\left\{\left(\theta_{1}, \ldots, \theta_{N}\right) \in T_{N} \mid \sum_{k=1}^{N} \cos \theta_{k}=0, \sum_{k=1}^{N} \sin \theta_{k}=0\right\} .
$$


The volume of $B_{0}$ is the one associated with the restriction of the metric $g=\sum_{1}^{3} d \theta^{2}$ to $B_{0}$. For a chain of $N=3$, using the formula (21) and a direct integration, we now compute the volume of $B_{0}$. There are basically two types of configurations, $P_{1}$ (Figure 1(A.a)) and $P_{2}$ (Figure 1(A.b)), characterized by the set of angle $S_{1}$ given by

$$
\begin{aligned}
& \theta_{2}=\theta_{1}+2 \pi / 3, \\
& \theta_{3}=\theta_{1}+4 \pi / 3
\end{aligned}
$$

and the set of angle $S_{2}$ given by

$$
\begin{aligned}
& \theta_{2}=\theta_{1}+4 \pi / 3, \\
& \theta_{3}=\theta_{1}+2 \pi / 3 .
\end{aligned}
$$

The $\epsilon$-neighborhoods $S_{1}(\epsilon), S_{2}(\epsilon)$ of $S_{1}$ and $S_{2}$, respectively, for small $\epsilon$, have two connected components which are approximately elliptic cylinders (Figure 2(a)). The metric induced on $S_{1}, S_{2}$ by the metric $g$ is

$$
d s_{g}^{2}=d \theta_{1}^{2}+d \theta_{2}^{2}+d \theta_{3}^{2}=3 d \theta_{1}^{2} .
$$

$B_{0}$ is a one dimensional manifold, the length of which is

$$
\left|B_{0}\right|=2 \int_{0}^{2 \pi} \sqrt{3} d \theta_{1}=4 \pi \sqrt{3} .
$$

The factor 2 accounts for the two configurations. Using (21), we obtain for the MFPT

$$
\left\langle\tau_{\epsilon, 3}\right\rangle=\frac{\pi}{D \sqrt{3}} \log \left(\frac{1}{\epsilon}\right)+\mathcal{O}(1) .
$$

We compare the analytical formula (34) obtained for uniformly distributed angles (equilibrium distribution) with Brownian simulations using the parameters $\epsilon=0.1$ and $D=0.01$ (50000 runs). We found with the analytical formula that $\left\langle\tau_{\epsilon, 3}\right\rangle=417$, while the Brownian simulations give the approximation result $\left\langle\tau_{\epsilon, 3}^{\text {num }}\right\rangle=450 \pm 7$. This result improves by decreasing the size $\epsilon$. In Appendix A, we present a direct method to estimate the zero order term, using a matching asymptotic approximation.

3.3. Estimating the MFLT for $N=4$. To estimate the MFLT For $N=4$, we first classify the closed sets of configurations $\left(\theta_{1}, \theta_{2}, \theta_{3}, \theta_{4}\right)$ by the condition

$$
\left|\exp \left(i \theta_{1}\right)+\exp \left(i \theta_{2}\right)+\exp \left(i \theta_{3}\right)+\exp \left(i \theta_{4}\right)\right|^{2}=0,
$$

which leads to

$$
\begin{aligned}
\cos \left(\theta_{2}-\theta_{1}\right) & +\cos \left(\theta_{3}-\theta_{1}\right)+\cos \left(\theta_{4}-\theta_{1}\right)+\cos \left(\theta_{3}-\theta_{2}\right) \\
& +\cos \left(\theta_{4}-\theta_{2}\right)+\cos \left(\theta_{4}-\theta_{3}\right)=-2 .
\end{aligned}
$$

There three sets of closed configurations $\left(S_{1}, S_{2}, S_{3}\right)$ (Figure 1 (C.a, b, and c), respectively) are parameterized as

$$
\begin{array}{ll}
S_{1}:\left\{\left(\theta_{1}, \theta_{2}, \theta_{1}+\pi, \theta_{2}+\pi\right) \mid\right. & \left.0 \leq \theta_{1}, \theta_{2} \leq 2 \pi\right\}, \\
S_{2}:\left\{\left(\theta_{1}, \theta_{2}, \theta_{2}+\pi, \theta_{1}+\pi\right) \mid\right. & \left.0 \leq \theta_{1}, \theta_{2} \leq 2 \pi\right\}, \\
S_{3}:\left\{\left(\theta_{1}, \theta_{1}+\pi, \theta_{3}, \theta_{3}+\pi\right) \mid\right. & \left.0 \leq \theta_{1}, \theta_{2} \leq 2 \pi\right\} .
\end{array}
$$


These configurations are invariant under the $S^{1}$-action $\left(\theta_{1}, \theta_{2}\right) \rightarrow\left(\theta_{1}+\varphi, \theta_{2}+\varphi\right)$, where $0 \leq \varphi \leq 2 \pi$. $S_{i}$ is a two dimensional manifold. Using the previous parametrization, we now calculate the volume of $B_{0}$. The metric induced on $S_{1}$ (or any other) is

$$
d s^{2}=2 d \theta_{1}^{2}+2 d \theta_{2}^{2}
$$

and det $g=4$. The volume of $B_{0}$ is thus (all three have the same volume)

$$
\left|B_{0}\right|=3 \int_{0}^{2 \pi} \int_{0}^{2 \pi} \sqrt{\operatorname{det} g} d \theta_{1} d \theta_{2}=24 \pi^{2} .
$$

The factor 3 accounts for the volume for $S 2, S 3$. Finally, using (21), the MFLT is

$$
\left\langle\tau_{\epsilon, 4}\right\rangle=\frac{\pi}{3 D} \log \left(\frac{1}{\epsilon}\right)+\mathcal{O}(1) .
$$

The section of $B_{\epsilon}$ by the 3-plane orthogonal to $(1,1,1)$ is depicted in Figure 2(b): for $\epsilon \ll 1, B_{\epsilon}$ consists of tubes with varying elliptic cross-section, connecting bulbs. There are three different bulb orientations associated with the different configurations (see (36)). In addition, $B_{\epsilon}$ has two connected components when $N=3$, while it has only one when $N=4$. In that case, any closed configuration can be continuously deformed into any other. Finally, using the same diffusion constant and $\epsilon$ as in the previous case $(N=3)$, we compare the analytical formula (39) with Brownian simulations. We find that $\left\langle\tau_{\epsilon, 4}\right\rangle=241$, while from the Brownian simulations we get the approximation $\left\langle\tau_{\epsilon, 4}^{\text {num }}\right\rangle=300 \pm 4$.

4. The MFLT for large $N$. Although the previous analysis is quite interesting, it was not possible to extend it to $N>4$. Thus, to study the dependency of the MFLT as a function of $N$, we use a different approach based on approximating the stochastic equations for large $N$. We start by deriving a stochastic equation for $\boldsymbol{R}_{N}(t)$ : using Itô's formula for $e^{i \theta_{k}(t)}[12]$,

$$
d e^{i \theta_{k}(t)}=i e^{i \theta_{k}(t)} d \theta_{k}(t)-D e^{i \theta_{k}(t)} d t
$$

Thus, by summing relations (40),

$$
d \boldsymbol{R}_{N}(t)=-D \boldsymbol{R}_{N}(t) d t+b d \tilde{\boldsymbol{W}}_{N}(t)
$$

where the noise term is described by

$$
d \tilde{\boldsymbol{W}}_{N}(t)=i \sum_{k=1}^{N} e^{i \theta_{k}(t)} d \theta_{k}(t) .
$$

We shall now show that the noise source $\tilde{\boldsymbol{W}}_{N}(t)$ can be approximated by a Brownian motion. Indeed, as $N$ goes to infinity, $\tilde{\boldsymbol{W}}_{N}(t)$ converges asymptotically to a Brownian motion [21] in the distribution sense. To start with, the first moment of $\tilde{\boldsymbol{W}}_{N}$ is zero. We have

$$
d \tilde{\boldsymbol{W}}_{N}(s)=\left(d \tilde{W}_{N}^{1}(s), d \tilde{W}_{N}^{2}(s)\right),
$$


where

$$
\begin{aligned}
& d \tilde{W}_{N}^{1}(s)=-\sum_{k=1}^{N} \sin \theta_{k}(t) d \theta_{k}(t), \\
& d \tilde{W}_{N}^{2}(s)=\sum_{k=1}^{N} \cos \theta_{k}(t) d \theta_{k}(t) .
\end{aligned}
$$

We shall now estimate the variance of $d \tilde{\boldsymbol{W}}_{N}(s)$. We estimate the quantities

$$
\begin{aligned}
& \int_{0}^{t}\left\langle d \tilde{W}_{N}^{1}(s), d \tilde{W}_{N}^{1}(s)\right\rangle=\int_{0}^{t} \sum_{j, k=1}^{N} \sin \theta_{j}(s) \sin \theta_{k}(s)\left\langle d \theta_{j}(s) d \theta_{k}(s)\right\rangle \\
& \int_{0}^{t}\left\langle d \tilde{W}_{N}^{2}(s), d \tilde{W}_{N}^{2}(s)\right\rangle=\int_{0}^{t} \sum_{j, k=1}^{N} \cos \theta_{j}(s) \cos \theta_{k}(s)\left\langle d \theta_{j}(s) d \theta_{k}(s)\right\rangle \\
& \int_{0}^{t}\left\langle d \tilde{W}_{N}^{1}(s), d \tilde{W}_{N}^{2}(s)\right\rangle=\int_{0}^{t} \sum_{j, k=1}^{N} \sin \theta_{j}(s) \cos \theta_{k}(s)\left\langle d \theta_{j}(s) d \theta_{k}(s)\right\rangle .
\end{aligned}
$$

We have $\left\langle d \theta_{j}(s) d \theta_{k}(s)\right\rangle=2 D \delta_{j, k} d s$. Taking the expectation,

$$
\begin{aligned}
E\left(\int_{0}^{t}\left\langle d \tilde{W}_{N}^{1}(s), d \tilde{W}_{N}^{1}(s)\right\rangle\right) & =2 D E\left(\int_{0}^{t} \sum_{k=1}^{N} \sin ^{2}\left(\theta_{k}(s)\right)\right) d s \\
& =2 N D E\left(\int_{0}^{t} \sin ^{2}\left(\theta_{1}(s)\right)\right) d s
\end{aligned}
$$

and

$$
\begin{aligned}
E\left(\sin ^{2}\left(\theta_{1}(t)\right)\right) d s & =\frac{1-e^{-4 D t}}{2} \\
\int_{0}^{t} E\left(\sin ^{2}\left(\theta_{1}(t)\right)\right) d s & =\frac{t}{2}+\frac{1-e^{-4 D t}}{8 D} .
\end{aligned}
$$

Thus

$$
E\left(\int_{0}^{t}\left\langle d \tilde{W}_{N}^{1}(s), d \tilde{W}_{N}^{1}(s)\right\rangle\right)=2 N D\left(\frac{t}{2}+\frac{1-e^{-4 D t}}{8 D}\right) \approx N D t
$$

and

$$
E\left(\int_{0}^{t}\left\langle d \tilde{W}_{N}^{2}(s), d \tilde{W}_{N}^{2}(s)\right\rangle\right)=2 N D\left(\frac{t}{2}+\frac{1+e^{-4 D t}}{8 D}\right) \approx N D t,
$$

the third coupling integral being zero. We conclude that for large times, the integrals in (51) and (52) can be approximated by $N D t$. The MFLT corresponds to the end point of the long polymer ( $N$ large) that enters into a small domain around the fixed end. Under this condition, we approximate the noise term $d \tilde{W}_{N}^{i}$ by a Brownian motion in both components with a diffusion diffusion constant $N D$. Finally, to explore the dependency of the MFLT as function of $N$, we will approximate the stochastic 
equation (41) by the Ornstein-Uhlenbeck process (some further details can be found in $[21])$

$$
d \boldsymbol{R}_{N}(t)=-D \boldsymbol{R}_{N}(t) d t+\sqrt{2 D N} b d \boldsymbol{W}(t),
$$

where $\boldsymbol{W}$ is a two dimensional Brownian motion of variance one. To evaluate the dependency on $N$ of the MFLT, we will now consider the dynamics of (53) in a ring of exterior and interior (small) radii $N b$ and $\epsilon$, respectively. The inner boundary $(r=\epsilon)$ is absorbing while we impose a Neumann boundary condition on the external boundary: The MFLT is thus the solution of the equation

$$
\begin{aligned}
N D b^{2} \Delta u-D\langle X, \nabla u\rangle & =-1 \quad \text { on } D(0, N b), \\
u(P) & =0 \quad \text { for }|P|=\epsilon b, \\
\frac{\partial u}{\partial n}(P) & =0 \quad \text { for }|P|=N b,
\end{aligned}
$$

where $X$ is the radial vector, and $D(0, N b)$ is the disk centered at the origin of radius $R=N b$. We are looking for a radially symmetric solution. Equation (55) in polar coordinate

$$
\begin{aligned}
N D b^{2}\left(u^{\prime \prime}+\frac{u^{\prime}}{r}\right)-D r u^{\prime} & =-1 \text { on } D(0, N b), \\
u(\epsilon) & =0 \\
u^{\prime}(N b) & =0
\end{aligned}
$$

is solved directly:

$$
u(r)=\frac{1}{D} \log \left(\frac{r}{\epsilon b}\right)-\frac{1}{D} e^{\frac{-N}{2}} \int_{\epsilon b}^{r} \frac{e^{\frac{v^{2}}{2 N b^{2}}}}{v} d v
$$

When the initial configuration of the rod-polymer is a straight segment $(r=R)$, the MFLT is

$$
u(R)=\frac{1}{D} \log \left(\frac{R}{\epsilon}\right)-\frac{1}{D} e^{\frac{-N}{2}} \int_{\epsilon b}^{R} \frac{e^{\frac{v^{2}}{2 N b^{2}}}}{v} d v .
$$

Integrating by parts and using Laplace's method for $N$ large, we get

$$
u(R=N b) \approx \frac{1}{D} \log \left(\frac{1}{\epsilon}\right)\left(1-e^{-\frac{N}{2}+\frac{\epsilon^{2}}{2 N}}\right)+\frac{1}{D} \log (N)\left(1-e^{(\epsilon-N)}\right) .
$$

Finally,

$$
\left\langle\tau_{\epsilon}\right\rangle=\frac{1}{D} \log \left(\frac{N}{\epsilon}\right)+\mathcal{O}_{\epsilon, \mathcal{N}}(1)
$$

Three regimes appear, depending on whether $N$ is $\gg \ll$ or $\approx \frac{1}{\epsilon}$. When $N \gg \frac{1}{\epsilon}$, then dependency in $\log (N)$ is dominant. 
4.1. The MFLT for an initial uniform distribution. To get the MFLT starting from a uniform distribution, we average $u(r)$ over all initial positions. We have

$$
\frac{1}{D} \int_{\varepsilon}^{R} \log \left(\frac{r}{\epsilon b}\right) r d r=\frac{R^{2}}{2 D}\left[\log \left(\frac{R}{\epsilon b}\right)-\frac{1}{2}\right]+\frac{(\epsilon b)^{2}}{4} ;
$$

for simplicity, we shall write in the following $\epsilon$ instead of $\epsilon b$. Using integration by parts,

$$
\mathrm{I}=\int_{\epsilon}^{R} \int_{\epsilon}^{r} r \frac{\exp \left(p v^{2}\right)}{v} d v d r=\int_{\epsilon}^{R} \frac{R^{2}-v^{2}}{2} \frac{\exp \left(p v^{2}\right)}{v} d v,
$$

where $p=\left(2 N b^{2}\right)^{-1}$, with $0<p \leq \frac{1}{2 b^{2}}$. Now

$$
\begin{aligned}
\mathrm{I} & =\int_{\epsilon}^{R} \frac{R^{2}}{2} \frac{\exp \left(p v^{2}\right)}{v} d v-\int_{\epsilon}^{R} \frac{v}{2} \exp \left(p v^{2}\right) d v, \\
\int_{\epsilon}^{R} \frac{v}{2} \exp \left(p v^{2}\right) d v & =\frac{\exp \left(p R^{2}\right)-\exp \left(p \epsilon^{2}\right)}{4 p} .
\end{aligned}
$$

Setting $w=v^{2}, u=p w$,

$$
\int_{\epsilon}^{R} \frac{\exp \left(p v^{2}\right)}{v} d v=\frac{1}{2} \int_{\epsilon^{2}}^{R^{2}} \frac{\exp (p w)}{w} d w=\frac{1}{2} \int_{p \epsilon^{2}}^{p R^{2}} \frac{\exp u}{u} d u .
$$

We shall estimate this last integral when $p R^{2} \rightarrow+\infty$ and $p \epsilon^{2} \rightarrow 0$ :

$$
\int_{p \epsilon^{2}}^{p R^{2}} \frac{\exp u}{u} d u=\int_{2}^{p R^{2}} \frac{\exp u}{u} d u+\int_{p \epsilon^{2}}^{2} \frac{\exp u}{u} d u \text {. }
$$

Applying two integrations by parts

$$
\begin{aligned}
& \int_{2}^{p R^{2}} \frac{\exp u}{u} d u=\left.\frac{\exp u}{u}\right|_{2} ^{p R^{2}}-\left.\frac{\exp u}{u^{2}}\right|_{2} ^{p R^{2}}+\int_{2}^{p R^{2}} \frac{2 \exp u}{u^{3}} d u \\
& \int_{2}^{p R^{2}} \frac{\exp u}{u} d u=\exp p R^{2}\left[\frac{1}{p R^{2}}-\frac{1}{p^{2} R^{4}}\right]+\int_{2}^{p R^{2}} \frac{2 \exp u}{u^{3}} d u+\mathcal{O}(1) .
\end{aligned}
$$

On the interval $[2,+\infty)$, the function $\frac{2 \exp u}{u^{3}}$ is increasing. Hence,

$$
\int_{2}^{p R^{2}} \frac{2 \exp u}{u^{3}} d u \leq \frac{2 \exp p R^{2}}{p^{2} R^{4}}
$$

Finally,

$$
\int_{2}^{p R^{2}} \frac{\exp u}{u} d u=\frac{\exp p R^{2}}{p R^{2}}\left(1+\mathcal{O}\left(\frac{1}{p R^{2}}\right)\right)+\mathcal{O}(1) .
$$

On the other hand,

$$
\int_{p \epsilon^{2}}^{2} \frac{\exp u}{u} d u=\log \frac{1}{p \epsilon^{2}}+\mathcal{O}(1) .
$$


Then

$$
\int_{p \epsilon^{2}}^{p R^{2}} \frac{\exp u}{u} d u=\frac{\exp p R^{2}}{p R^{2}}\left(1+\mathcal{O}\left(\frac{1}{p R^{2}}\right)\right)+\log \frac{1}{p \epsilon^{2}}+\mathcal{O}(1) .
$$

Substituting the expressions (61) and (60) into the expression for I (59),

$$
\mathrm{I}=\frac{\exp p R^{2}}{4 p}\left(1+\mathcal{O}\left(\frac{1}{p R^{2}}\right)\right)+\frac{R^{2}}{2} \log \frac{1}{p \epsilon^{2}}+\mathcal{O}\left(R^{2}\right)
$$

and

$$
\begin{aligned}
\int_{\epsilon}^{R} u(r) r d r= & \frac{R^{2}}{2 D} \log \left(\frac{R}{\epsilon}\right) \\
& -\frac{1}{D} \exp \left(\frac{-N}{2}\right)\left[\frac{\exp p R^{2}}{4 p}\left(1+\mathcal{O}\left(\frac{1}{p R^{2}}\right)\right)+\frac{R^{2}}{2} \log \frac{1}{p \epsilon^{2}}\right]+\mathcal{O}\left(R^{2}\right) .
\end{aligned}
$$

We conclude that the MFLT for a chain starting with a uniform distribution is

$$
\left\langle\tau_{\epsilon}\right\rangle=\frac{1}{\pi R^{2}} \int_{\epsilon b}^{R} u(r) r d r \approx \frac{1}{2 \pi D} \log \left(\frac{N}{\epsilon}\right)+\mathcal{O}_{\epsilon, \mathcal{N}}(1) .
$$

This asymptotic formula is different from the one obtained for a chain initially straight (formula (57)). We conclude that the leading order term depends on the initial configuration of the polymer. This is quite surprising compared to the narrow escape formula, derived for a Brownian particle, where the leading order term does not depend on the initial configuration [11]. It seems that the long rod-polymer chain has a memory of the initial configuration.

4.2. The MFLT for a polymer starting with an initial OrnsteinUhlenbeck equilibrium distribution. We now estimate the MFLT starting from an equilibrium distribution and use this computation to compare the analytical formula with our Brownian simulation (Figure 3). Because the end monomer satisfies the Ornstein-Uhlenbeck equation (53), the equilibrium distribution depends only on the distance $r$ to the origin and its solution of the forward Fokker-Planck equation

$$
N D b^{2} \Delta p+D \nabla \cdot(X \nabla p)=0
$$

and is given by

$$
p(r)=\operatorname{Pr}\left\{R_{N}=r\right\}=\frac{1}{2 \pi N b^{2}} e^{-\frac{r^{2}}{2 N b^{2}}} .
$$

To calculate the MFLT starting from the Ornstein-Uhlenbeck equilibrium distribution, we average $u(r)$ with respect to the density $p(r)$ :

$$
\left\langle\tau_{\epsilon}\right\rangle=\frac{1}{D} \int_{0}^{2 \pi} \int_{\epsilon}^{N b} \frac{r d r d \theta}{2 \pi N b^{2}} e^{-\frac{r^{2}}{2 N b^{2}}}\left[\log \left(\frac{r}{\epsilon}\right)-e^{-\frac{N}{2}} \int_{\epsilon}^{r} \frac{\exp \left(v^{2} / 2 N b^{2}\right)}{v} d v\right] .
$$

We have

$$
\mathrm{I}_{1}=\frac{1}{N b^{2}} \int_{\epsilon}^{N b} e^{-\frac{r^{2}}{2 N b^{2}}} \log \left(\frac{r}{\epsilon}\right) r d r
$$



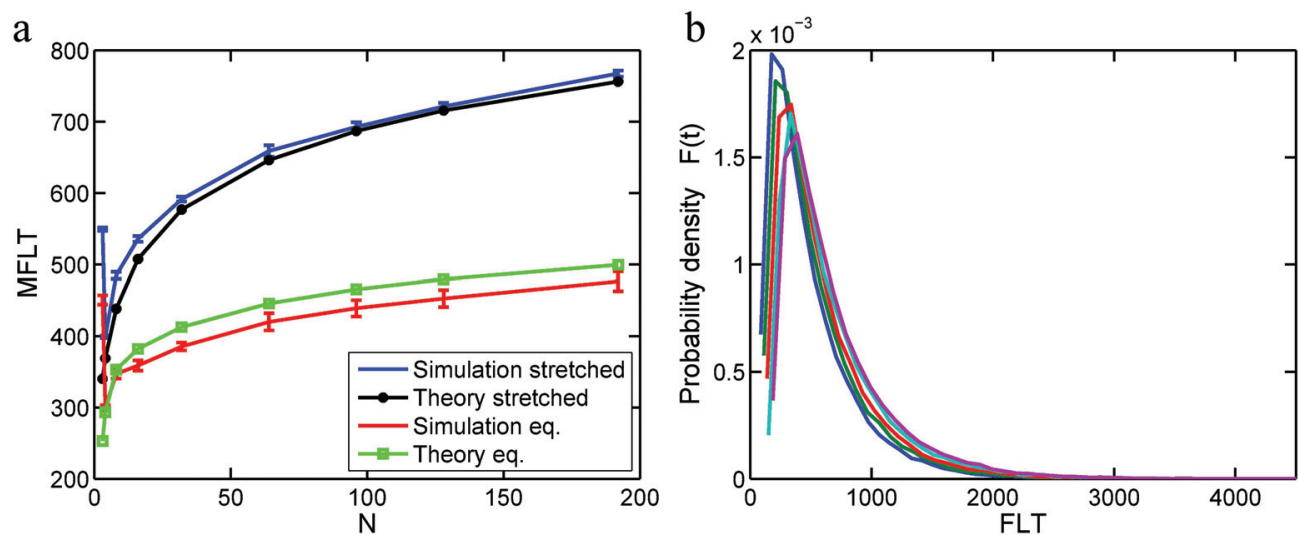

FIG. 3. Brownian simulations of the MFLT in dimension 2. The initial configuration of the polymer is stretched (blue and black) or at equilibrium (red and green) ((64)), and the first monomer is fixed at the origin. (a) MFLT of the polymer for several values of $N$, with $\epsilon=0.1$ and $D=0.01$. We use 50000 independent runs. We compare here the Brownian simulation (red and blue) with the analytical solutions (green and black) of (57) (initially stretch polymer) and (71) (initially at equilibrium). For large $N$, the dependence of the mean time is logarithmic in $N$ as predicated by our analysis. (b) Probability density function of the first looping time $F(t)=\frac{d}{d t} \operatorname{Pr}\{\tau<t\}$ for several polymer lengths $N=8,16,32,64,96$ (going left to right). At long times, the curve-decay exponentially [11]. Color is available only in the online version.

and using the change of variables $u=r^{2} / 2 N b^{2}$,

$$
\mathrm{I}_{1}=\int_{\epsilon^{2} / 2 N b^{2}}^{N / 2} e^{-u} \log \left(\frac{\sqrt{2 N b^{2} u}}{\epsilon}\right) d u .
$$

The integral can be divided into two terms

$$
\int_{\epsilon^{2} / 2 N b^{2}}^{N / 2} e^{-u} \log \left(\frac{\sqrt{2 N b^{2}}}{\epsilon}\right) d u=\log \left(\frac{\sqrt{2 N b^{2}}}{\epsilon}\right)\left(e^{\epsilon^{2} / 2 N b^{2}}-e^{-N / 2}\right)
$$

and

$$
\frac{1}{2} \int_{\epsilon^{2} / 2 N b^{2}}^{N / 2} e^{-u} \log (u) d u
$$

When $N \gg 1$ and $\epsilon \ll 0$, the second integral is bounded from above

$$
\begin{aligned}
\frac{1}{2} \int_{\epsilon^{2} / 2 N b^{2}}^{N / 2} e^{-u} \log (u) d u & <\int_{\epsilon^{2} / 2 N b^{2}}^{N / 2} u e^{-u} d u \\
& =e^{\epsilon^{2} / 2 N b^{2}}\left(\frac{\epsilon^{2}}{2 N b^{2}}+1\right)-e^{-N / 2}\left(1+\frac{N}{2}\right) \cong \mathcal{O}(1)
\end{aligned}
$$

and from below

$$
\begin{aligned}
\int_{\epsilon^{2} / 2 N b^{2}}^{N / 2} e^{-u} \log (u) d u> & -\int_{\epsilon^{2} / 2 N b^{2}}^{N / 2} u^{-1 / 2} e^{-u} d u=-\int_{0}^{N / 2} u^{-1 / 2} e^{-u} d u \\
& +\int_{0}^{\epsilon^{2} / 2 N b^{2}} u^{-1 / 2} e^{-u} d u=\Gamma\left(\frac{3}{2}, \epsilon^{2} / 2 N b^{2}\right)-\Gamma\left(\frac{3}{2}, \frac{N}{2}\right) .
\end{aligned}
$$


For $N \gg 1$ and $\epsilon \ll 1, \Gamma\left(\frac{3}{2}, \epsilon^{2} / 2 N b^{2}\right) \approx \Gamma(3 / 2)=\frac{\sqrt{\pi}}{2}$ while $\Gamma\left(\frac{3}{2}, \epsilon^{2} / 2 N b^{2}\right)$ goes to zero. Now we evaluate the second integral

$$
\mathrm{I}_{2}=\frac{1}{N b^{2}} \int_{\epsilon}^{N b} e^{-\frac{r^{2}}{2 N b^{2}}} r d r \int_{\epsilon}^{r} \frac{\exp \left(v^{2} / 2 N b^{2}\right)}{v} d v .
$$

Integrating by parts, we get

$$
\begin{aligned}
\mathrm{I}_{2} & =-\left.e^{-\frac{r^{2}}{2 N b^{2}}} \int_{\epsilon}^{r} \frac{\exp \left(v^{2} / 2 N b^{2}\right)}{v} d v\right|_{\epsilon} ^{N b}+\int_{\epsilon}^{N b} \frac{1}{r} d r \\
& =-e^{N / 2} \int_{\epsilon}^{N b} \frac{\exp \left(v^{2} / 2 N b^{2}\right)}{v} d v+\log \left(\frac{N b}{\epsilon}\right) .
\end{aligned}
$$

We shall estimate the last integral

$$
\begin{aligned}
\mathrm{I}_{3} & =\int_{\epsilon}^{N b} \frac{\exp \left(v^{2} / 2 N b^{2}\right)}{v} d v=\int_{\epsilon^{2} / 2 N b^{2}}^{N / 2} \frac{e^{u}}{u} d u \\
& =\int_{\epsilon^{2} / 2 N b^{2}}^{3} \frac{e^{u}}{u} d u+\int_{3}^{N / 2} \frac{e^{u}}{u} d u .
\end{aligned}
$$

Further integrations by parts leads to

$$
\begin{aligned}
& \int_{3}^{N / 2} \frac{e^{u}}{u} d u=\left.\frac{e^{u}}{u}\right|_{3} ^{N / 2}-\left.\frac{e^{u}}{u^{2}}\right|_{3} ^{N / 2}+\int_{3}^{N / 2} \frac{2 e^{u}}{u^{3}} d u, \\
& \int_{3}^{N / 2} \frac{e^{u}}{u} d u=e^{N / 2}\left[\frac{2}{N}-\frac{4}{N^{2}}\right]+\int_{3}^{N / 2} \frac{2 e^{u}}{u^{3}} d u+\mathcal{O}(1) .
\end{aligned}
$$

On the interval $[3,+\infty)$, the function $\frac{2 e^{u}}{u^{3}}$ is increasing. Hence,

$$
\int_{3}^{N / 2} \frac{2 e^{u}}{u^{3}} d u \leq \frac{8 e^{N / 2}}{N^{2}} .
$$

Finally,

$$
\int_{3}^{N / 2} \frac{e^{u}}{u} d u=\frac{2 e^{N / 2}}{N}\left(1+\mathcal{O}\left(\frac{1}{N^{2}}\right)\right)+\mathcal{O}(1) .
$$

On the other hand,

$$
\int_{\epsilon^{2} / 2 N b^{2}}^{3} \frac{e^{u}}{u} d u=\log \left(\frac{2 N b^{2}}{\epsilon^{2}}\right)+\mathcal{O}(1) .
$$

Finally, we obtain that

$$
\begin{aligned}
\left\langle\tau_{\epsilon}\right\rangle= & \frac{1}{D} \log \left(\frac{\sqrt{2 N b^{2}}}{\epsilon}\right)\left(e^{\epsilon^{2} / 2 N b^{2}}-e^{-N / 2}\right) \\
& -\frac{1}{D} e^{-N / 2}\left[\log \left(\frac{N b}{\epsilon}\right)-\frac{2 e^{N / 2}}{N}\left(1+\mathcal{O}\left(\frac{1}{N^{2}}\right)\right)+\mathcal{O}(1)\right]+\mathcal{O}(1) .
\end{aligned}
$$

Thus the MFLT starting from the equilibrium distribution (64) is given by

$$
\left\langle\tau_{\epsilon}\right\rangle=\frac{1}{D} \log \left(\frac{\sqrt{2 N}}{\epsilon}\right)+\mathcal{O}\left(\frac{1}{N}\right)+\mathcal{O}(1),
$$

where the leading order term depends on the initial configuration. 


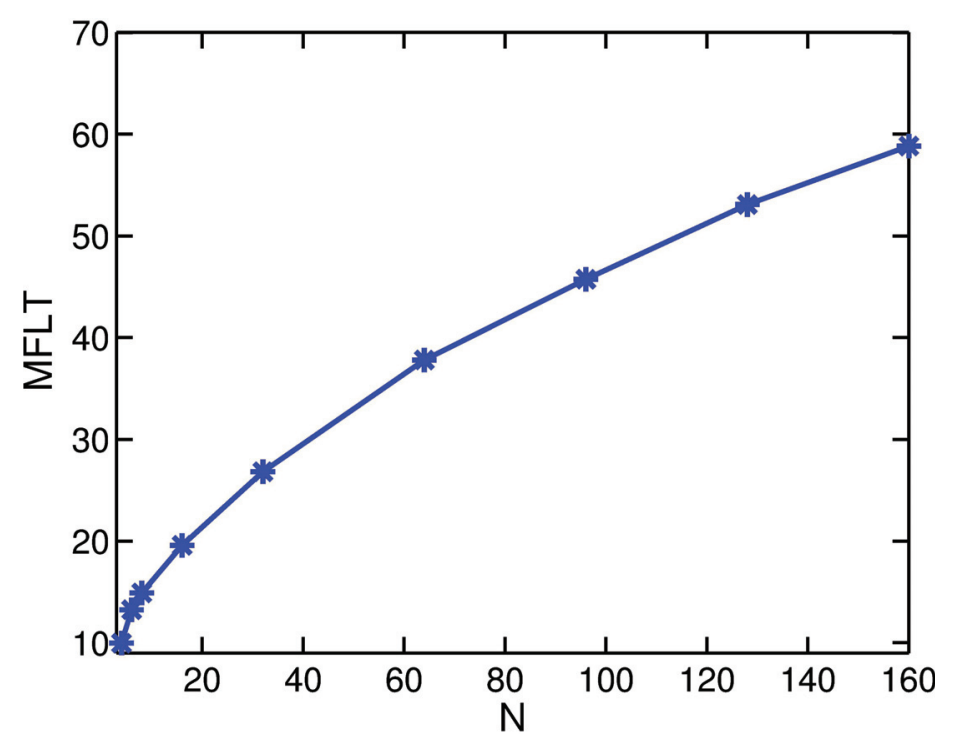

FIG. 4. Numerical simulation for the MFLT in dimension 3. The initial configuration is distributed according to (64). We plot the MFLT for several values of $N$. The parameters are $\epsilon=0.1, D=1$. For large $N$, a fitting estimate gives $\left\langle\tau_{\epsilon}\right\rangle \propto N^{\alpha}$, where $\alpha=0.48 \pm 0.02$.

5. Discussion. Using a rod-like type model, we obtained asymptotic estimates for the MFLT of a polymer in dimension 2. Interestingly, the MFLT depends critically on the size $\epsilon$ and on the length of the polymer. Surprisingly, the mean time to loop is larger for a chain with $N=3$ than that of a chain with $N=4$. The reason for this behavior is that $N=3$ is the only instance in which the tubular neighborhood $B_{\epsilon}$ has two connected components. Unfortunately, the present approach for large $N$ cannot be easily extended to dimension 3 because we are missing a general simple representation of rotations that would lead to a stochastic equation of motion. However, using Brownian simulations, generalizing (4) to three dimensions, we anticipated (Figure 4) a mean looping time power law $N^{1 / 2}$. The reason for this scaling law is due to the motion of the monomers on the surface of the three dimensional unit sphere, but the precise derivation of this scaling law is under investigation.

It is not easy to compare our results with the rest of the literature in polymer looping. Indeed, our model (see (4)-(6)) belongs to a different universality class compared to the classical polymer models $[3,17,6,20]$. The dynamics of the Rouse model and more complicated models used in these works are frequently analyzed by using the normal mode transformation [4]. The coupling between monomers gives rise to a dynamics that can be characterized by anomalous diffusion [8]. In contrast, in our polymer model, coupling between the monomers appears only in their position (see (4)) but not between angles (see (6)), leading to an approximation by an OrnsteinUhlenbeck process.

Finally, we have found here that the leading order for large $N$ term depends on the initial configuration, showing that for long polymers the process has a long memory. This is probably due to the correlation of the polymer motion. Furthermore, while the simulation results for the MFLT starting from a stretched configuration match the analytical result very well, there is a difference when the starting configuration is at equilibrium. We have found (Figure 3 ) that the simulation and analytical results differ by a constant term, suggesting that the next order term in $\epsilon$ in the expansion 
of the MFLT is of order 1. It would interesting to obtain an analytical expression. Although we use here a crude model for polymer, the present analysis is very general and can be adapted to other models, such as the Rouse model or the semiflexible chain model [13], which better account for DNA dynamics.

Appendix A. In this appendix, we present a description of the manifold $B_{0}$ and a method for computing the first order term for $N=3$.

A.1. Description of the motion space. In this section we describe the configuration spaces representing the possible shapes of the polymers (open or closed). We have represented the polymers as a linkage of a given number $N$ of rigid links of the same fixed length $l$. Hence in this representation the polymer having the nodes $\left(P_{0}, P_{1}, \ldots, P_{N}\right)$ is represented by a ordered sequence $\left(v_{1}, v_{2}, \ldots, v_{N}\right)$ of elements of $S^{1}(l)$, the circle in the plane having center 0 and radius $l: v_{j}$ is the vector $\overrightarrow{P_{j-1} P_{j}}$. Hence the space of all configurations of polymers is the torus $T^{N}(l)=$ $S^{1}(l) \times S^{1}(l) \times \cdots \times S^{1}(l)$. The configurations of closed polymers form the $N$ subspace $B_{0}$ of $T^{N}(l)$ :

$$
B_{0}=\left\{\left(v_{1}, v_{2}, \ldots, v_{N}\right) \in T^{N}(l) \mid v_{1}+v_{2}+\cdots+v_{N}=0\right\} .
$$

We summarize our result in the following proposition.

Proposition A.1. (a) If $N$ is odd, $B_{0}$ is a submanifold of $T^{N}(l)$.

(b) If $N$ is even, $B_{0}$ is a real subanalytic set, the singular set of which, Sing $B_{0}$, is the disjoint union of the orbits of the $\frac{N !}{\left(\frac{N}{2}\right) !}$ points $\left\{\left(\sigma_{1}, \ldots, \sigma_{N}\right) \mid \sigma_{j} \in\{ \pm 1\}, \sigma_{1}+\right.$ $\left.\cdots+\sigma_{N}=0\right\}$ under the action $\Delta$.

Proof. $\left(v_{1}, \ldots, v_{N}\right)$ is a singular point of $M_{0}^{N}$ iff for all $i, j, 1 \leq i<j \leq N$, the imaginary part of $v_{i} \bar{v}_{j}$ is null. Because $v_{1}+\cdots+v_{N}=0$, this can happen only if $N$ is even and only for the points defined in (b). It would be very interesting to identify $B_{0}$ with some known manifolds or to give a more informative description of it. This is possible in some cases (small $N$ ). Also there are some simple procedures which allow us to reduce the study of $B_{0}$ to that of manifolds of smaller dimension. This is presented in the following section.

Study of the polymer configuration space.

(a) When $N=3$, then $B_{0}^{3}$ is the orbit under the action of $\Delta$ of the point $\left(1, \exp \left(\frac{2 \pi i}{3}\right), \exp \left(\frac{4 \pi i}{3}\right)\right)$.

(b) $N=4, \Phi_{4}$ maps $B_{0}^{4}$ onto the antidiagonal $A D=\{(z,-z) \mid z \in \mathbb{D}\}$. $\mathcal{O}$ is the dense open subset $\left\{\left(v_{1}, v_{2}, v_{3}, v_{4}\right) \mid v_{1} \neq \pm v_{2}, v_{3} \neq \pm v_{4}\right\}$ of $T^{4}$. If we set $v_{j}=$ $l \exp \left(\theta_{j} i\right), 1 \leq j \leq 4$, then

$$
\Phi_{4}\left(v_{1}, v_{2}, v_{3}, v_{4}\right)=\left(\cos \left[\frac{\theta_{1}-\theta_{2}}{2}\right] \exp \left(i \frac{\theta_{1}+\theta_{2}}{2}\right), \cos \left[\frac{\theta_{3}-\theta_{4}}{2}\right] \exp \left(i \frac{\theta_{3}+\theta_{4}}{2}\right)\right) .
$$

The polar coordinates on $\mathbb{D}(l) \times \mathbb{D}(l)$ are $\left(r_{1}, \varphi_{1}, r_{2}, \varphi_{2}\right)$. The natural metric of $T^{4}$ is $d s_{T}^{2}=\sum_{n=1}^{4} d \theta_{n}^{2}$. The image by $\Phi_{4}$ of the restriction of the image $\left(\Phi_{4}\right)_{*}\left[d s_{T}^{2}\right]$ to the open subset $\Phi_{4}(\mathcal{O})$ of $\mathbb{D} \times \mathbb{D}$ is

$$
g=\sum_{j=1}^{2}\left[\frac{\left(d r_{j}\right)^{2}}{1-r_{j}^{2}}+d \varphi_{j}^{2}\right] .
$$


Hence the restriction $\tilde{g}$ of this metric to the antidiagonal $A D \cap \Phi_{4}(\mathcal{O})$ is

$$
\tilde{g}=4\left[\frac{(d r)^{2}}{1-r^{2}}+d \varphi^{2}\right] .
$$

The area of $A D$ as a surface in $\mathbb{D} \times \mathbb{D}$ for the concept of area derived from the metric $\left(\Phi_{4}\right)_{*}\left[d s_{T}^{2}\right]$ is the same as that of $A D \cap \Phi_{4}(\mathcal{O})$ because $A D-A D \cap \Phi_{4}(\mathcal{O})$ is reduced to the point $(0,0)$. Hence this area is given by

$$
\int_{0}^{2 \pi} \int_{0}^{1} \frac{4 d r d \varphi}{\sqrt{1-r^{2}}}=4 \pi^{2}
$$

Because $\Phi_{4}$ is a covering mapping of degree 4 , the area of $M_{0}^{4}$ for the concept of area derived from the metric $d s_{T}^{2}$ is $16 \pi^{2}$.

(c) A useful reduction. We are going to define a smooth mapping

$$
\Phi_{N}: T^{N} \rightarrow\left\{\begin{array}{l}
\mathbb{D}^{\frac{N}{2}}, N \text { even, } \\
\mathbb{D}^{\frac{N-1}{2}} \times S^{1}, N \text { odd },
\end{array}\right.
$$

i where $\mathbb{D}$ is the closed disc of center 0 and radius 1 :

$$
\Phi_{N}\left(v_{1}, \ldots, v_{N}\right)=\left\{\begin{array}{l}
\left(\frac{v_{1}+v_{2}}{2}, \frac{v_{3}+v_{4}}{2}, \ldots, \frac{v_{N-1}+v_{N}}{2}\right), \\
\left(\frac{v_{1}+v_{2}}{2}, \frac{v_{3}+v_{4}}{2}, \ldots \frac{v_{N-2} v_{N-1}}{2}, v_{N}\right) .
\end{array}\right.
$$

Let us note that if $e^{i \theta_{1}}, e^{i \theta_{2}} \in S^{1}$, then $\frac{e^{i \theta_{1}}+e^{i \theta_{2}}}{2}=\cos \left(\frac{\theta_{1}-\theta_{2}}{2}\right) e^{i \frac{\theta_{1}+\theta_{2}}{2}}$. Let $\mathcal{B}^{\prime}$ be the subset of $T^{N}$ :

$$
\begin{aligned}
\mathcal{B}^{\prime} & =\left\{\left(v_{1}, \ldots, v_{N}\right) \in T^{N} \mid \exists ! n,\right. \\
1 & \leq n \leq\left\{\begin{array}{l}
N-1, N \text { even, } \\
N-2, N \text { odd, }
\end{array} \text { such that } v_{n}= \pm v_{n+1}\right\}
\end{aligned}
$$

Proposition A.2. The restriction of $\Phi_{N}$ to $T^{N}-\mathcal{B}_{0}$ is a covering of order $2^{\frac{N}{2}}$ if $N$ is even (resp., $2^{\frac{N-1}{2}}$ if $N$ is odd) of the manifold $\mathbb{D}^{\frac{N}{2}}-\mathcal{B}_{0}$, where $\mathcal{B}_{0}=$ $\left\{\left(w_{1}, \ldots, w_{\frac{N}{2}}\right)\left|\exists n, 1 \leq n \leq \frac{N}{2},\right| w_{n} \mid \in\{0,1\}\right.$ (resp., $\left\{\left(w_{1}, \ldots, w_{\frac{N-1}{2}}, v_{N}\right) \mid \exists n, 1 \leq n \leq\right.$ $\left.\left.\frac{N-1}{2},\left|w_{n}\right| \in\{0,1\}\right\}\right)$.

$\Phi_{N}$ has a section

$$
\Psi_{N}:\left\{\begin{array}{l}
\mathbb{D}^{\frac{N}{2}}-\mathcal{B}_{0} \\
\mathbb{D}^{\frac{N-1}{2}} \times S^{1}-\mathcal{B}_{0}
\end{array} \rightarrow T^{N}\right.
$$

To define it we shall introduce a mapping $\psi: \mathbb{D}_{2}-\{0\} \rightarrow S^{1} \times S^{1}$. Let $w \in \mathbb{D}-\{0\}$. Then there exists a unique $\alpha \in\left[0, \frac{\pi}{2}\right]$ such that $|w|=\cos (\alpha)$. Let $\zeta=\frac{w}{\cos (\alpha)} \in S^{1}$. Then we define $\psi(w)=\left(\zeta e^{\frac{-i \alpha}{2}}, \zeta e^{i \frac{\alpha}{2}}\right)$. Using $\psi$,

$$
\Psi_{N}=\left\{\begin{array}{l}
\frac{\underbrace{\psi \times \cdots \times \psi}_{\frac{N}{2} \text { times }}}{\psi \times \cdots \times \psi} \times I d_{S 1} \\
\underbrace{\psi \times \cdots}_{\frac{N-1}{2} \text { times }} .
\end{array}\right.
$$


A.2. Second term in the asymptotic expansion of the MFLT for $N=3$ : Direct application of the matching asymptotic method. We shall here present an application of the matching asymptotic method [23, 22, 24, 9] to compute the leading and the zero order terms in the asymptotic expansion of the MFLT when $N=3$. The boundary $B_{\epsilon}$ is defined by

$$
\left|\exp \left(i \theta_{1}\right)+\exp \left(i \theta_{2}\right)+\exp \left(i \theta_{3}\right)\right|^{2}=\epsilon^{2}
$$

which simplifies to

$$
\cos \left(\theta_{2}-\theta_{1}\right)+\cos \left(\theta_{3}-\theta_{1}\right)+\cos \left(\theta_{3}-\theta_{2}\right)=\frac{\epsilon^{2}-3}{2} .
$$

In the variables $u=\theta_{2}-\theta_{1}$ and $v=\theta_{3}-\theta_{1}$, we obtain

$$
\cos (u)+\cos (v)+\cos (u-v)=\frac{\epsilon^{2}-3}{2} .
$$

The two closed configurations for $\epsilon=0$ in the $u, v$ plan correspond to the points $P_{1}=(u=2 \pi / 3, v=4 \pi / 3)$ and $P_{2}=(u=4 \pi / 3, v=2 \pi / 3)$. Thus the boundary $B_{\epsilon}$ consists precisely of the union of two narrow cylinders $C_{1}$ and $C_{2}$ in the torus $T_{3}$ associated, respectively, with the points $P_{1}$ and $P_{2}$. To further characterize these cylinders, we use a Taylor expansion of (81). At $P_{1}$, the cylindrical section is

$$
S_{1}=\left\{(u, v) \mid\left(u-\frac{1}{2} v\right)^{2}+\frac{3}{4}\left(v-\frac{4 \pi}{3}\right)^{2}+o\left(u^{2}+v^{2}\right)=\epsilon^{2}\right\},
$$

which is approximately an ellipse with a major axis of length $a \epsilon$ with $a=\frac{2}{\sqrt{3}}$ and a minor axis of length $b \epsilon$ with $b=1$. The axis of $C_{1}$ consists of a line $L_{1}$, defined by $\tilde{P}_{1}=(X, Y)_{1}=(0,4 \pi / 3)$, where $X=u-\frac{1}{2} v, Y=v$. Similarly, the second ellipse is given by

$$
S_{2}=\left\{(u, v) \mid\left(v-\frac{1}{2} u\right)^{2}+\frac{3}{4}\left(u-\frac{4 \pi}{3}\right)^{2}+o\left(u^{2}+v^{2}\right)=\epsilon^{2}\right\},
$$

and in the variables, $X=v-\frac{1}{2} u, Y=u$, the axis of $C_{2}$ is a line $L_{2}$, centered at $\tilde{P}_{2}=(X, Y)_{2}=(4 \pi / 3,0)$, parallel to $L_{1}$, in the direction of the unit vector $\frac{1}{\sqrt{3}}(1,1,1)$.

We now construct the solution of the MFLT using the method of matching asymptotic expansions. Because the absorbing boundary consists of two parallel cylinders $C_{1}$ and $C_{2}$ of elliptic section (see (82)-(83)), inside a three-dimensional torus, we first construct an inner solution using the $u, v$ variables. In the rescale transverse coordinates, $\eta_{1}=\frac{u}{a}, \eta_{2}=\frac{v}{b}$. The solution $[23,9]$ near $P_{1}$ is invariant by translation and thus reduces to a two dimensional problem. The inner solution is given by

$$
u(\boldsymbol{x}) \approx-\frac{1}{2 \pi} \log \frac{\left|\boldsymbol{x}-\tilde{P}_{1}\right|_{g_{1}}}{\epsilon},
$$

where $g_{1}$ is the metric induced by ellipse 1 . Similarly, near $P_{2}$,

$$
u(\boldsymbol{x}) \approx-\frac{1}{2 \pi} \log \frac{\left|\boldsymbol{x}-\tilde{P}_{2}\right|_{g_{2}}}{\epsilon}
$$

and $g_{2}$ is the metric induces by the ellipse 2 . 
To solve the MFLT to the cylinder, we use the transversal Green's function $G$, the solution of

$$
\begin{aligned}
\Delta G(\boldsymbol{x}, \boldsymbol{y}) & =\frac{1}{|S|}-\delta(\boldsymbol{y}) \quad \text { for } \boldsymbol{x} \in T_{2}, \\
\int_{\Omega} G(\boldsymbol{x}, \boldsymbol{y}) d \boldsymbol{x} & =0
\end{aligned}
$$

where $T_{2}$ is the plan section $(u, v)$ in the torus and $|S|$ the transversal surface area. Near the cylinder $C_{1}, C_{2}$, the solution is invariant along the cylindrical axis. We have

$$
G_{1}\left(\boldsymbol{x}, \tilde{P}_{1}\right)=-\frac{1}{2 \pi} \log \frac{\left|\boldsymbol{x}-\tilde{P}_{1}\right|_{g_{1}}}{\epsilon}+R_{1}\left(\boldsymbol{x}, \tilde{P}_{1}\right) .
$$

Similarly, the solution $G_{2}$ near the cylinder 2 is

$$
G_{2}\left(\boldsymbol{x}, \tilde{P}_{2}\right)=-\frac{1}{2 \pi} \log \frac{\left|\boldsymbol{x}-\tilde{P}_{2}\right|_{g_{2}}}{\epsilon}+R_{2}\left(\boldsymbol{x}, \tilde{P}_{2}\right),
$$

where $R_{1}, R_{2}$ are the regular parts of the Green's function [18]. The general solution in the torus thus is the sum

$$
u(\boldsymbol{x})=A_{1} G_{1}\left(\boldsymbol{x}, \tilde{P}_{1}\right)+A_{2} G_{2}\left(\boldsymbol{x}, \tilde{P}_{2}\right)+C \quad \text { for } \boldsymbol{x} \in T-C_{1} \cup C_{2},
$$

where $A_{1}, A_{2}, C$ are three constants to be determined. $C$ is the average of $u$ over the section and thus over the torus. To determine the constants, we first choose a point $\boldsymbol{y}_{1} \in \partial C_{1}$

$$
\begin{aligned}
0= & u\left(\boldsymbol{y}_{1}\right)=A_{1}\left(-\frac{1}{2 \pi} \log \frac{\left|\boldsymbol{y}_{1}-\tilde{P}_{1}\right|_{g_{1}}}{\epsilon}+R_{1}\left(\boldsymbol{y}_{1}, \tilde{P}_{1}\right)\right) \\
& +A_{2}\left(-\frac{1}{2 \pi} \log \frac{\left|\boldsymbol{y}_{1}-\tilde{P}_{2}\right|_{g_{2}}}{\epsilon}+R_{2}\left(\boldsymbol{y}_{1}, \tilde{P}_{2}\right)\right)+C
\end{aligned}
$$

that is,

$$
0 \approx A_{1} R_{1}\left(\tilde{P}_{1}, \tilde{P}_{1}\right)+A_{2}\left(-\frac{1}{2 \pi} \log \frac{\left|\tilde{P}_{1}-\tilde{P}_{2}\right|_{g_{2}}}{\epsilon}+R_{2}\left(\tilde{P}_{1}, \tilde{P}_{2}\right)\right)+C .
$$

For $\boldsymbol{y}_{2} \in C_{2}$,

$$
\begin{aligned}
0= & u\left(\boldsymbol{y}_{2}\right)=A_{1}\left(-\frac{1}{2 \pi} \log \frac{\left|\boldsymbol{y}_{2}-\tilde{P}_{1}\right|_{g_{1}}}{\epsilon}+R_{1}\left(\boldsymbol{y}_{2}, \tilde{P}_{1}\right)\right) \\
& +A_{2}\left(-\frac{1}{2 \pi} \log \frac{\left|\boldsymbol{y}_{2}-\tilde{P}_{2}\right|_{g_{2}}}{\epsilon}+R_{2}\left(\boldsymbol{y}_{2}, \tilde{P}_{2}\right)\right)+C
\end{aligned}
$$

and

$$
0 \approx A_{1}\left(-\frac{1}{2 \pi} \log \frac{\left|\tilde{P}_{2}-\tilde{P}_{1}\right|_{g_{1}}}{\epsilon}+R_{1}\left(\tilde{P}_{2}, \tilde{P}_{1}\right)\right)+A_{2} R_{2}\left(\tilde{P}_{2}, \tilde{P}_{2}\right)+C
$$


Finally, the conservation of flux (taking the Laplacian of (90) in $T-C_{1} \cup C_{2}$ ) and the definition of the Green's function lead to

$$
A_{1}+A_{2}=-|S| \text {. }
$$

Finally, the solution of the linear system of equations is with

$$
R_{1}\left(\boldsymbol{y}_{1}, \tilde{P}_{1}\right)=R_{111}, R_{1}\left(\boldsymbol{y}_{2}, \tilde{P}_{1}\right)=R_{121}, R_{2}\left(\boldsymbol{y}_{1}, \tilde{P}_{2}\right)=R_{212}, R_{2}\left(\boldsymbol{y}_{2}, \tilde{P}_{2}\right)=R_{222},
$$

and we obtain

$$
C=-|S| \frac{\left(\frac{1}{4 \pi^{2}} \log \frac{d_{1}}{\epsilon} \log \frac{d_{2}}{\epsilon}-\frac{1}{2 \pi} \log \frac{d_{1}}{\epsilon} R_{121}-\frac{1}{2 \pi} \log \frac{d_{2}}{\epsilon} R_{212}+R_{212} R_{121}-R_{111} R_{212}\right)}{\left(\frac{1}{2 \pi} \log \frac{d_{1} d_{2}}{\epsilon^{2}}-R_{121}-R_{212}+R_{111}+R_{222}\right)}
$$

and

$$
\begin{aligned}
& A_{1}=C \frac{R_{111}+\frac{1}{2 \pi} \log \frac{d_{1}}{\epsilon}+R_{121}}{\left(\frac{1}{2 \pi} \log \frac{d_{1} d_{2}}{\epsilon^{2}}-R_{121}-R_{212}+R_{111}+R_{222}\right)}, \\
& A_{1}=C \frac{R_{222}+\frac{1}{2 \pi} \log \frac{d_{2}}{\epsilon}+R_{212}}{\left(\frac{1}{2 \pi} \log \frac{d_{1} d_{2}}{\epsilon^{2}}-R_{121}-R_{212}+R_{111}+R_{222}\right)} .
\end{aligned}
$$

The computation for the area of $S$ is done in the plane perpendicular to the vector $(1,1,1)$ (Figure 2(a)). The surface $S$ is periodic on this plane and it can be computed directly by using the formula for a two dimensional polyhedron, formed by the six cylinders in the figure. The area of a polyhedron is given by $A=\frac{3 \sqrt{3}}{2} t^{2}$, where $t=\frac{\sqrt{8} \pi}{3}$ is the length of an edge which is also the distance between $P_{1}$ and $P_{2}$. Each of the six cylinders contributes $1 / 3$ to each polyhedron (as it is at the intersection of three polyhedrons), and we have two full cylinders in each $S$. Thus, the surface is $S=A \frac{6}{2 \cdot 3}=\frac{4 \pi^{2}}{\sqrt{3}}$. Finally, we recover from (97) the leading order term in formula (34)

$$
C=\frac{S}{4 \pi} \log \frac{1}{\epsilon}+\mathcal{O}(1)=\frac{\pi}{\sqrt{3}} \log \frac{1}{\epsilon}+\mathcal{O}(1) .
$$

The zero order term depends on the regular part of the Green's function associated with the different metrics for each ellipse.

\section{REFERENCES}

[1] L. Breiman, Probability, Addison-Wesley, Reading, MA, 1968.

[2] I. Chavel and E. Feldman, Spectra of manifolds less a small domain, Duke Math. J., 56 (1988), pp. 399-414.

[3] M. DoI, Diffusion-controlled reaction of polymers, Chem. Phys., 9 (1975), pp. 455-466.

[4] M. Doi And S. F. Edwards, The Theory of Polymer Dynamics, Clarendon Press, Oxford, UK, 1986.

[5] B. Friedman and B. O'Shaughnessy, Short time behavior and universal relations in polymer cyclization, J. Phys. II, 1 (1991), pp. 471-486.

[6] B. Friedman and B. O'Shaughnessy, Theory of intramolecular reactions in polymeric liquids, Macromolecules, 26 (1993), pp. 4888-4898.

[7] D. Holcman and Z. Schuss, Escape through a small opening: Receptor trafficking in a synaptic membrane, J. Stat. Phys., 117 (2004), pp. 975-1014.

[8] Y. Kantor and M. Kardar, Anomalous diffusion with absorbing boundary, Phys. Rev. E, 76 (2007), 061121. 
[9] T. Kolokolnikov, M. Titcombe, and M. WARD, Optimizing the fundamental Neumann eigenvalue for the Laplacian in a domain with small traps, European J. Appl. Math., 16 (2005), pp. 161-200.

[10] R. Pastor, R. Zwanzig, And A. Szabo, Diffusion limited first contact of the ends of a polymer: Comparison of theory with simulation, J. Chem. Phys., 105 (1996), pp. 3878-3882.

[11] Z. Schuss, A. Singer, And D. Holcman, The narrow escape problem for diffusion in cellular microdomains, Proc. Natl. Acad. Sci. USA, 104 (2007), pp. 16098-16103.

[12] Z. Schuss, Theory and Applications of Stochastic Differential Equations, Wiley Ser. Probab. Stat.. John Wiley and Sons, New York, 1980.

[13] R. Shusterman, S. Alon, T. Gavrinyov, and O. Krichevsky, Monomer dynamics in doubleand single-stranded DNA polymers, Phys. Rev. Lett., 92 (2004), 048303.

[14] A. Singer, D. Holcman, and B. Eisenberg, Narrow escape I, J. Stat. Phys., 122 (2006), pp. $437-463$.

[15] A. Singer, Z. Schuss, And D. Holcman, Narrow escape II, J. Stat. Phys., 122 (2006), pp. 465489.

[16] A. Singer, Z. Schuss, And D. Holcman, Narrow escape III, J. Stat. Phys., 122 (2006), pp. 491509.

[17] I. Sokolov, Cyclization of a polymer: First-passage problem for a non-Markovian process, Macromolecules, 90 (2003), 080601.

[18] R. Straube, M. J. Ward, and M. Falcke, Reaction rate of small diffusing molecules on a cylindrical membrane, J. Stat. Phys., 129 (2007), pp. 377-405.

[19] A. Szabo, K. Schulten, and Z. Schulten, First passage time approach to diffusion controlled reactions, J. Chem. Phys., 72 (1980), pp. 4350-4357.

[20] N. M. Toan, G. Morrison, C. Hyeon, and D. Thirumalai, Cyclization of Rouse chains at long- and short-time scales, J. Chem. Phys. B., 112 (2008), pp. 6094-6106.

[21] S. Vakroudis, M. Yor, AND D. Holcman, The mean first rotation time of a planar polymer, J. Stat. Phys., 143 (2011), pp. 1074-1095.

[22] M. J. Ward, W. D. Henshaw, and J. B. Keller, Summing logarithmic expansions for singularly perturbed eigenvalue problems, SIAM J. Appl. Math., 53 (1993), pp. 799-828.

[23] M. J. WARD AND J. B. Keller, Strong localized perturbations of eigenvalue problems, SIAM J. Appl. Math., 53 (1993), pp. 770-798.

[24] M. WARd And E. VAn De Velde, The onset of thermal runaway in partially insulated or cooled reactors, IMA J. Appl. Math., 48 (1992), pp. 53-85.

[25] G. Wilemski and M. Fixman, Diffusion-controlled intrachain reactions of polymers. II Results for a pair of terminal reactive groups, J. Chem. Phys., 60 (1974), pp. 878-890.

[26] G. Wilemski and M. Fixman, Diffusion-controlled intrachain reactions of polymers. I Theory, J. Chem. Phys., 60 (1974), pp. 866-877. 